\title{
Engineering E. coli for simultaneous glucose-xylose utilization during methyl ketone production
}

\author{
Xi Wang ${ }^{1,2}$, Ee-Been Goh ${ }^{1,2}$ and Harry R. Beller ${ }^{1,2,3^{*}}$ (1)
}

\begin{abstract}
Background: We previously developed an E. coli strain that overproduces medium-chain methyl ketones for potential use as diesel fuel blending agents or as flavors and fragrances. To date, the strain's performance has been optimized during growth with glucose. However, lignocellulosic biomass hydrolysates also contain a substantial portion of hemicellulose-derived xylose, which is typically the second most abundant sugar after glucose. Commercialization of the methyl ketone-producing technology would benefit from the increased efficiency resulting from simultaneous, rather than the native sequential (diauxic), utilization of glucose and xylose.

Results: In this study, genetic manipulations were performed to alleviate carbon catabolite repression in our most efficient methyl ketone-producing strain. A strain engineered for constitutive expression of $x y \mid F$ and $x y / A$ (involved in xylose transport and metabolism) showed synchronized glucose and xylose consumption rates. However, this newly acquired capability came at the expense of methyl ketone titer, which decreased fivefold. Further efforts were made to improve methyl ketone production in this strain, and we found that two strategies were effective at enhancing methyl ketone titer: (1) chromosomal deletion of pgi (glucose-6-phosphate isomerase) to increase intracellular NADPH supply and (2) downregulation of CRP (CAMP receptor protein) expression by replacement of the native RBS with an RBS chosen based upon mutant library screening results. Combining these strategies resulted in the most favorable overall phenotypes for simultaneous glucose-xylose consumption without compromising methyl ketone titer at both 1 and $2 \%$ total sugar concentrations in shake flasks.
\end{abstract}

Conclusions: This work demonstrated a strategy for engineering simultaneous utilization of $\mathrm{C}_{6}$ and $\mathrm{C}_{5}$ sugars in $E$. coli without sacrificing production of fatty acid-derived compounds.

Keywords: Carbon catabolite repression, Methyl ketones, NADPH, CAMP receptor protein, Metabolic engineering

\section{Background}

Medium-chain (e.g., $\mathrm{C}_{11}-\mathrm{C}_{15}$ ) methyl ketones are among the fatty acid-derived compounds that have been developed recently for potential application as diesel blending agents [1-5]. A methyl ketone biosynthetic pathway in Escherichia coli that has attained $40 \%$ of maximum theoretical yield with glucose as the sole carbon source includes the following features: (a) overproduction of $\beta$-ketoacyl-coenzyme A (CoA) thioesters achieved by

\footnotetext{
*Correspondence: HRBeller@lbl.gov

1 Joint BioEnergy Institute (JBEI), 5885 Hollis St., Emeryville, CA 94608, USA Full list of author information is available at the end of the article
}

modification of the $\beta$-oxidation pathway (via overexpression of native FadB and a heterologous acyl-CoA oxidase from Micrococcus luteus) and (b) overexpression of a native thioesterase (FadM) that is effective at hydrolyzing $\beta$-ketoacyl-CoA thioesters to $\beta$-keto acids, which are the immediate precursors of methyl ketones [2]. Performance of the methyl ketone-producing strain for this technology has thus far been optimized during growth with glucose [2], which is typically the dominant sugar in biomass hydrolysates. However, biomass hydrolysates also contain a substantial portion of hemicellulose-derived xylose (typically, the second most abundant sugar after glucose). Commercialization of the methyl ketone-producing 
technology would benefit from the increased efficiency resulting from simultaneous utilization of glucose and xylose [6].

A challenge in cultivating $E$. coli in growth medium containing both glucose and xylose is diauxic (phased, non-simultaneous) growth, whereby glucose must be depleted before other sugars, such as xylose, can be metabolized [7]. The underlying mechanism for diauxic growth is carbon catabolite repression (CCR), which is primarily mediated by components of the phosphoenolpyruvate (PEP): carbohydrate phosphotransferase (PTS) system. The glucose-specific EII complex of the PTS system consists of the permease, EIIBC ${ }^{\text {Glc }}$ (encoded by $p t s G$ ), and EIIA ${ }^{\text {Glc }}$ (encoded by $c r r$ ), which has a primary role in modulating carbohydrate metabolism in $E$. coli. During glucose transport, EIIA ${ }^{\text {Glc }}$ is dephosphorylated, which prevents either the import of non-glucose sugars or their subsequent metabolism, and as a consequence, bacterial cells are devoid of the inducer for the corresponding operons; this is known as inducer exclusion [8]. One consequence of dephosphorylated EIIA ${ }^{\text {Glc }}$ is a decrease in levels of cyclic AMP (cAMP), which is produced from ATP by adenylate cyclase (activated by phosphorylated EIIA ${ }^{\text {Glc }}$ ). Lower levels of cAMP in turn limit the availability of cAMP-CRP, the complex between cAMP and CRP (cAMP receptor protein). The expression of genes that are involved in the catabolism of sugars other than glucose generally requires the CAMP-CRP complex and, consequently, is repressed in the presence of glucose. In addition, the arabinose transcriptional regulator (AraC) suppresses the xylose-catabolism genes $x y l A B$ and $x y l F G H$ by inhibiting the xylose transcriptional activator (XylR), which constitutes the second layer of CCR [9].

Multiple strategies have been proposed for engineering simultaneous hexose-pentose metabolism in E. coli by mitigating CCR, such as inactivation of pts $G$, mutation of regulatory genes, and constitutive expression of key genes in pentose metabolism [10-16]. However, few studies have applied such strategies for mitigating CCR to production of fatty acid-based biofuels [17]. Challenges can be anticipated in combining metabolic strategies for simultaneous glucose-xylose utilization and methyl ketone overproduction, as changes in central carbon metabolism can substantially alter redox balance, which is needed for efficient conversion of carbon to targeted products [18].

In this study, we investigated the effects of engineering CCR mitigation into our best methyl ketone-overproducing E. coli strain (EGS1895 [2]). We chose to follow the CCR mitigation strategy recently described by Kim et al. [12], which was reported to offer advantages over other approaches, most notably, the engineered strains grow well on glucose, unlike some CCR-insensitive mutants defective in the glucose PTS system. Several rounds of engineering were required to optimize both (1) simultaneous glucose-xylose co-utilization and (2) methyl ketone production, as strategies targeting one of these outcomes often adversely affected the other. Ultimately, our results suggest the feasibility of engineering simultaneous utilization of glucose and xylose in E. coli along with substantial production of fatty acid-derived biofuels.

\section{Methods}

\section{Strains, plasmids, and reagents}

Escherichia coli strains and plasmids are listed in Table 1. Strains and plasmids along with their associated information (annotated GenBank-format sequence files) have been deposited in the public version of the JBEI Registry (https://public-registry.jbei.org; entries JPUB_009980 to JPUB_010000) and are physically available from the authors and/or addgene (http://www.addgene.org) upon request. Our previously developed methyl ketone-overproducing strain EGS1895 [2] was used as the control strain. Q5 High-Fidelity DNA Polymerase was used for all PCR reactions (New England Biolabs, Ipswich, MA). NEBuilder HiFi DNA Assembly Master Mix (New England Biolabs, Ipswich, MA) was used to assemble linear DNA fragments. Plasmid extractions were carried out by using QIAGEN miniprep kits (Valencia, CA). Oligonucleotide primers were synthesized by Integrated DNA Technologies, Inc. (San Diego, CA). DNA sequencing was completed by GENEWIZ (South Plainfield, NJ).

\section{Genetic manipulations and strain development}

All genome engineering was conducted by using the $\lambda$-Red recombination system with vectors pKD13, pKD46, and pCP20 [19, 20]. All primers used in this study are listed in Additional file 1: Table S1. CCR mitigation strategies developed by Kim et al. [12] (Table 2) were used to engineer the simultaneous utilization of glucose and xylose in strain EGS1895. Specifically, the synthetic constitutive promoters CP6 and CP25 [21] were used to replace native promoters for key genes in pentose transport (araFGH and $x y l F G H$ ) and catabolism ( $\operatorname{araBAD}$ and $x y l A B)$, respectively. The arabinose transcription factor ara $C$ was deleted from the chromosome. Additionally, the arabinose-proton symporter araE was inactivated and a point mutation was introduced in the $5^{\prime}$-flanking region of $x y l A\left(x y l A^{u p}\right)$. We also deleted araC from the methyl ketone-pathway plasmid pEG1675 [2]. By inserting an $a r a C$-free fragment between SpeI and AgeI restriction sites of pEG1675, a new plasmid, pXW1677, was created. The resultant strain with pXW1677 was named XW1044 (Tables 1, 2), and the two intermediate strains were named XW1014 and XW1024 (Tables 1, 2). 
Table 1 Strains and plasmids used in this study

\begin{tabular}{|c|c|c|}
\hline & Relevant characteristics & Source or reference \\
\hline \multicolumn{3}{|l|}{ Strains } \\
\hline EGS1405 & E. coli DH1; $\triangle$ fadE; $\triangle a c k A-p t a ; \triangle p o x B$ & [2] \\
\hline EGS1895 & EGS1405 with pEG1675 & {$[2]$} \\
\hline XW1003 & E. coli DH1; $\triangle$ fadE; $\triangle a c k A-p t a ; \triangle p o x B ; \Delta p t s G$ & This study \\
\hline XW1004 & XW1003 with pEG1675 & This study \\
\hline XW1013 & E. coli DH1; $\triangle$ fadE; $\triangle a c k A-p t a ; \triangle p o x B ; P_{C P 6}-x y\left|F ; P_{C P 25}-x y\right| A ; x y \mid A^{\text {up }}$ & This study \\
\hline XW1014 & XW1013 with pEG1675 & This study \\
\hline XW1018 & XW1013 with pXW1678 & This study \\
\hline XW1023 & 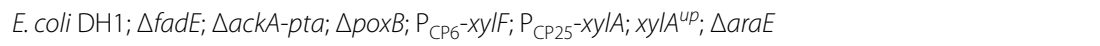 & This study \\
\hline XW1024 & XW1023 with pEG1675 & This study \\
\hline XW1043 & 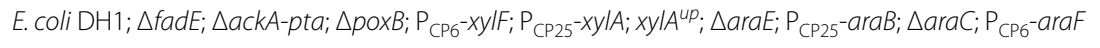 & This study \\
\hline XW1044 & XW1043 with pXW1677 & This study \\
\hline XW1053 & E. coli DH1; $\triangle$ fadE; $\triangle a c k A-p t a ; \Delta p o x B ; P_{C P 6}-x y \mid F ; P_{C P 25}-x y / A ; x y / A^{\text {up }} ; \Delta p g i$ & This study \\
\hline XW1054 & XW1053 with pEG1675 & This study \\
\hline XW1055 & XW1053 with pXW1678 & This study \\
\hline XW1063 & 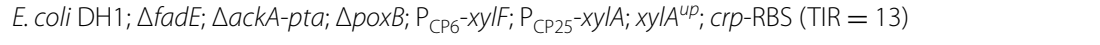 & This study \\
\hline XW1064 & XW1063 with pEG1675 & This study \\
\hline XW1073 & 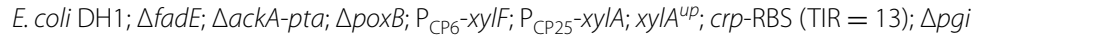 & This study \\
\hline XW1074 & XW1073 with pEG1675 & This study \\
\hline XW1075 & XW1073 with pXW1678 & This study \\
\hline \multicolumn{3}{|l|}{ Plasmids } \\
\hline pKD13 & $\lambda$-Red recombineering plasmid & [19] \\
\hline pKD46 & $\lambda$-Red recombineering plasmid & [19] \\
\hline pCP20 & $\lambda$-Red recombineering plasmid & [19] \\
\hline pEG1675 & 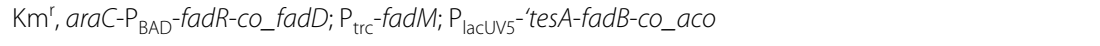 & [2] \\
\hline pXW1677 & pEG1675 with araC deleted & This study \\
\hline pXW1678 & $\mathrm{Km}^{r}$, araC-P $\mathrm{BAD}_{\mathrm{BA}}-$ fadR-CO_fadD; $\mathrm{P}_{\mathrm{tr}}$-fadM-maeB; $\mathrm{P}_{\text {lacuv5 }}{ }^{-}$tes A-fadB-co_aco & This study \\
\hline
\end{tabular}

Table 2 Strategies used for engineering simultaneous glucose-xylose utilization in EGS1895

\begin{tabular}{|c|c|c|c|c|c|}
\hline No. & Manipulation & Target sequence & XW1014 & XW1024 & XW1044 \\
\hline 1 & Replace promoter of xyIFGH & CP6 synthetic promoter & $+^{b}$ & + & + \\
\hline 2 & Replace promoter of $x y \mid A B$ & CP25 synthetic promoter & + & + & + \\
\hline 3 & $x y / A^{u p}$ mutation & $\mathrm{G}(+6) \rightarrow \mathrm{T}$ of $\mathrm{CP} 25$ & + & + & + \\
\hline 4 & araE inactivation & Truncated araE & & + & + \\
\hline 5 & Replace promoter of araFGH & CP6 synthetic promoter & & & + \\
\hline 6 & Replace promoter of araBAD & CP25 synthetic promoter & & & + \\
\hline 7 & Chromosomal deletion of araC & $\triangle a r a C$ (genome) & & & + \\
\hline 8 & araC deletion from pEG1675 & $\triangle a r a C$ (plasmid) & & & + \\
\hline
\end{tabular}

a Based on [12]

b + indicates that the specified genetic modification is present in this strain

For constructing the pXW1678 plasmid, the E. coli DH1 native $m a e B$ gene was cloned and inserted downstream of $f a d M$ at an SalI restriction site on pEG1675.

Studies were conducted to modulate and optimize the expression of the CRP by testing a set of crp ribosomal binding sites (RBS). To mutate the RBS for CRP, the RBS Library Calculator [22] was used to design RBS mutant library sequences and $\lambda$-Red recombineering was used to integrate the library mutants into the host genome. For RBS calculations, the $\lambda$-Red-generated 81-bp scar 
sequence (5'-ATTCCGGGGATCCGTCGACCTGCAGT TCGAAGTTCCTATTCTCTAGAAAGTATAGGAA CTTCGAAGCAGCTCCAGCCTACA-3') was used as the pre-sequence. Based on the native 35-bp RBS sequence for $c r p$ (5'-CTCTGGAGAAAGCTTATAACAGAGGATAACCGCGC-3'), a degenerate RBS sequence (5'-CTHTGGTGAAAGCTTATAACTGAGGMRAACCGCGT-3') was generated with a broad range of predicted translation initiation rates (TIR) for a total 12 variant sequences. RBS sequences and their predicted TIR values are listed in Additional file 1: Table S2. For reference, the native $c r p$ RBS sequence in $E$. coli DH1 was predicted to have a TIR value of 2441 au based on the RBS Calculator [23, 24].

\section{Media and culture conditions}

Lysogeny broth (LB) was used for routine cell growth and propagation. Kanamycin was added to the growth medium at a final concentration of $50 \mu \mathrm{g} \mathrm{mL}^{-1}$, when required. M9-MOPS minimal medium with $1 \%$ total sugars $\left(5 \mathrm{~g} \mathrm{~L}^{-1}\right.$ glucose and $5 \mathrm{~g} \mathrm{~L}^{-1}$ xylose) or $2 \%$ total sugars (10 $\mathrm{g} \mathrm{L}^{-1}$ glucose and $10 \mathrm{~g} \mathrm{~L}^{-1}$ xylose) as carbon sources was used for production experiments. The composition of M9-MOPS minimal medium followed the recipe previously described [2]. For production experiments, strains were first adapted in M9-MOPS minimal medium with $1 \%$ total sugars for 3 passages before being inoculated into production medium with glucose and xylose.

\section{Analysis of cell growth and sugar metabolism}

Cell growth was monitored by measuring optical density at $600 \mathrm{~nm}\left(\mathrm{OD}_{600}\right)$. Sugars were measured with an Agilent 1100 Series HPLC system, equipped with an Agilent 1200 Series refractive index detector (RID) (Agilent Technologies, CA) and Aminex HPX-87H ion-exclusion column (300-mm length, 7.8-mm internal diameter; BioRad Laboratories, Inc., Hercules, CA). The column temperature was $50{ }^{\circ} \mathrm{C}$, and $4 \mathrm{mM}$ sulfuric acid was used as the mobile phase with a flow rate of $0.6 \mathrm{~mL} \mathrm{~min}{ }^{-1}$ for $24 \mathrm{~min}$. The quantification of glucose and xylose was conducted by external standard calibration with authentic standards.

\section{Production and analysis of methyl ketones}

For methyl ketone production, strains were inoculated into $50 \mathrm{~mL}$ M9-MOPS minimal medium with $1 \%$ total sugars, or $25 \mathrm{~mL}$ with $2 \%$ total sugars, in $250-\mathrm{mL}$ shake flasks and cultured at $37^{\circ} \mathrm{C}$ with 200-rpm agitation. The starting $\mathrm{OD}_{600}$ during production was ca. 0.01. Gene expression was induced by adding $0.2 \mathrm{mM}$ IPTG and $1 \mathrm{mM}$ arabinose after $6 \mathrm{~h}$ of growth. Five $\mathrm{mL}$ of decane (Reagent-Plus $\geq 99 \%$ purity, Sigma-Aldrich, St. Louis, $\mathrm{MO})$ amended with perdeuterated tetracosane $\left(\mathrm{C}_{24} \mathrm{D}_{50}\right)$ and 3-tetradecanone (Sigma-Aldrich, St. Louis, MO) as internal standards was also added to the cultures during induction. The decane overlay was sampled for the measurement of methyl ketones; analysis by electron ionization gas chromatography-mass spectrometry (GC-MS) was conducted as previously described [3].

\section{Batch fermentation of strains EGS1895 and XW1075 in a 2-L bioreactor for methyl ketone production}

Batch fermentation was carried out in a 2-L bioreactor equipped with a Sartorius BIOSTAT B plus control unit for regulating dissolved oxygen (DO), $\mathrm{pH}$, and temperature. Frozen glycerol stocks of M9-MOPS-adapted cells were used to seed a test tube containing $5 \mathrm{~mL}$ of M9-MOPS minimal medium with $1 \%$ total sugars $\left(5 \mathrm{~g} \mathrm{~L}^{-1}\right.$ glucose and $5 \mathrm{~g} \mathrm{~L}^{-1}$ xylose) as previously described. After $30 \mathrm{~h}$ of growth, the cultures were diluted 1:250 into a 250-mL flat-bottom shake flask containing $50 \mathrm{~mL}$ of M9-MOPS minimal medium supplemented with $1 \%$ total sugars. This culture was grown for another $30 \mathrm{~h}$ as before and was used to inoculate $1.25 \mathrm{~L}$ of medium in the bioreactor. The medium was adapted from Korz et al. [25] and was composed of M9 salts $\left(6.8 \mathrm{~g} \mathrm{~L}^{-1} \mathrm{Na}_{2} \mathrm{HPO}_{4}, 3.0 \mathrm{~g} \mathrm{~L}^{-1}\right.$ $\mathrm{KH}_{2} \mathrm{PO}_{4}, 1.0 \mathrm{~g} \mathrm{~L}^{-1} \mathrm{NH}_{4} \mathrm{Cl}, 0.5 \mathrm{~g} \mathrm{~L}^{-1} \mathrm{NaCl}$ ) supplemented with $0.5 \mathrm{~g} \mathrm{~L}^{-1}$ of $\mathrm{MgSO}_{4} \cdot 7 \mathrm{H}_{2} \mathrm{O}, 0.18 \mathrm{~g} \mathrm{~L}^{-1}$ of $\mathrm{NH}_{4} \mathrm{Cl}$, $1.0 \mathrm{mg} \mathrm{L}{ }^{-1}$ thiamine, $10 \mathrm{nM}$ of $\mathrm{FeSO}_{4} \cdot 7 \mathrm{H}_{2} \mathrm{O}, 100 \mu \mathrm{M}$ $\mathrm{CaCl}_{2} \cdot 2 \mathrm{H}_{2} \mathrm{O}$, micronutrients as described previously [2], $10 \mathrm{~g} \mathrm{~L}^{-1}$ of glucose, $10 \mathrm{~g} \mathrm{~L}^{-1}$ of xylose, and $50 \mu \mathrm{g} \mathrm{mL}^{-1}$ of kanamycin. The temperature of the bioreactor was maintained at $37^{\circ} \mathrm{C}$ throughout the fermentation and the culture was maintained at pH 6.5 automatically by the addition of a $10 \mathrm{M}$ potassium hydroxide solution. The initial stir rate and airflow were set at $200 \mathrm{rpm}$ and $1 \mathrm{VVM}$ (volume of air per volume of liquid per minute), respectively. Dissolved oxygen was maintained above $40 \%$ of saturation via cascade control of adjustment of stirrer speed (up to $1600 \mathrm{rpm}$ ), followed by air-flow rate (up to $2.0 \mathrm{VVM}$ ). Cultures were induced with $1 \mathrm{mM}$ arabinose and $0.5 \mathrm{mM}$ IPTG at $6 \mathrm{~h}$ after initiation of batch phase. In addition, $150 \mathrm{~mL}$ of dodecane (Sigma-Aldrich, ReagentPlus $\geq 99 \%$ purity) amended with $3 \mathrm{mg} \mathrm{mL}^{-1}$ of 3-tetradecanone (Sigma-Aldrich) as an internal standard, was added into the bioreactors.

At selected times, $10-15-\mathrm{mL}$ samples were removed from the bioreactors via a syringe affixed to the sampling tube while the stirrer was still operating. Approximately $50 \mu \mathrm{L}$ of cell cultures were filtered through a $0.2-\mu \mathrm{m}$ syringe membrane filter directly into a vial for HPLC analysis and the rest of the cultures transferred to a $15-\mathrm{mL}$ Falcon tube. After allowing the samples to sit in the $15-\mathrm{mL}$ tube for $1 \mathrm{~min}$, the supernatant dodecane overlay was pipetted out into a 2-mL microcentrifuge tube and centrifuged at $21,130 \times g$ for $10 \mathrm{~min}$ to obtain a 
better-resolved aqueous-organic interface. The dodecane overlay was transferred into a glass vial and stored at $4{ }^{\circ} \mathrm{C}$ until GC-MS analysis.

\section{Results and discussion}

Engineering simultaneous glucose-xylose utilization in methyl ketone-overproducing strain EGS1895

We engineered several strains by manipulating key genes in pentose metabolism (XW1014, XW1024, and XW1044; Tables 1, 2) and evaluated their ability to simultaneously utilize glucose and xylose (Fig. 1). The control strain, EGS1895, presented a typical diauxic pattern in which xylose utilization began after glucose was fully depleted. In contrast, newly engineered strains displayed glucose-xylose co-utilization to varying degrees rather than a strict diauxic profile. Among these engineered strains, XW1014 (with constitutive expression of xylA and $x y l F$ plus a point mutation in the $x y l A$ promoter, $x y l A^{u p}$ ) showed the best performance for simultaneous utilization of glucose and xylose (Fig. 1). This strain had identical consumption rates for glucose and xylose at $1 \%$ sugar conditions, while a slight decrease in xylose consumption was observed at higher sugar concentration (2\%). The inactivation of araE (XW1024; Tables 1, 2) did not result in better sugar co-utilization than was observed for strain XW1014, nor did the manipulations made for strain XW1044 (alleviating AraC-mediated repression through four collective ara $C$-related manipulations, including ara $\mathrm{C}$ deletion from both the genome and plasmid as well as replacement of promoters for $\operatorname{araB}$ and araF). Although both strains XW1024 and XW1044 showed favorable simultaneous consumption rates of glucose and xylose at $1 \%$ sugar conditions, their xylose consumption dramatically decreased at higher sugar concentration (2\%).

In addition, because ptsG deficiency is a well-studied mechanism for mitigating CCR in E. coli [10], the glucose transporter EIIBC Glc encoded by ptsG was deleted from EGS1895 to investigate the effect on sugar co-utilization (strain XW1004; Table 1). Strain XW1004 did not display a better sugar co-utilization profile than strain XW1014 (Fig. 1).

Methyl ketone production was also investigated among these strains engineered for hexose-pentose co-utilization. Compared with the titer of the control strain EGS1895 ( $\left.690 \mathrm{mg} \mathrm{L}^{-1}\right)$, methyl ketone production was significantly reduced in all four modified strains (Fig. 2). The best performing strain for sugar co-utilization, XW1014, only produced $\sim 140 \mathrm{mg} \mathrm{L}^{-1}$ total methyl ketones (1\% total sugars), which is approximately fivefold lower than for strain EGS1895. Strains with more genetic manipulations produced even lower methyl ketone titers; for example, strains XW1024 and XW1044 produced $<60 \mathrm{mg} \mathrm{L}^{-1}$ methyl ketones. Although the $\Delta p t s G$ strain (XW1004) showed the highest methyl ketone titer among these four strains, its diminished glucose utilization was not optimal and it was not pursued further. Despite its relatively low methyl ketone titer, strain XW1014 had the
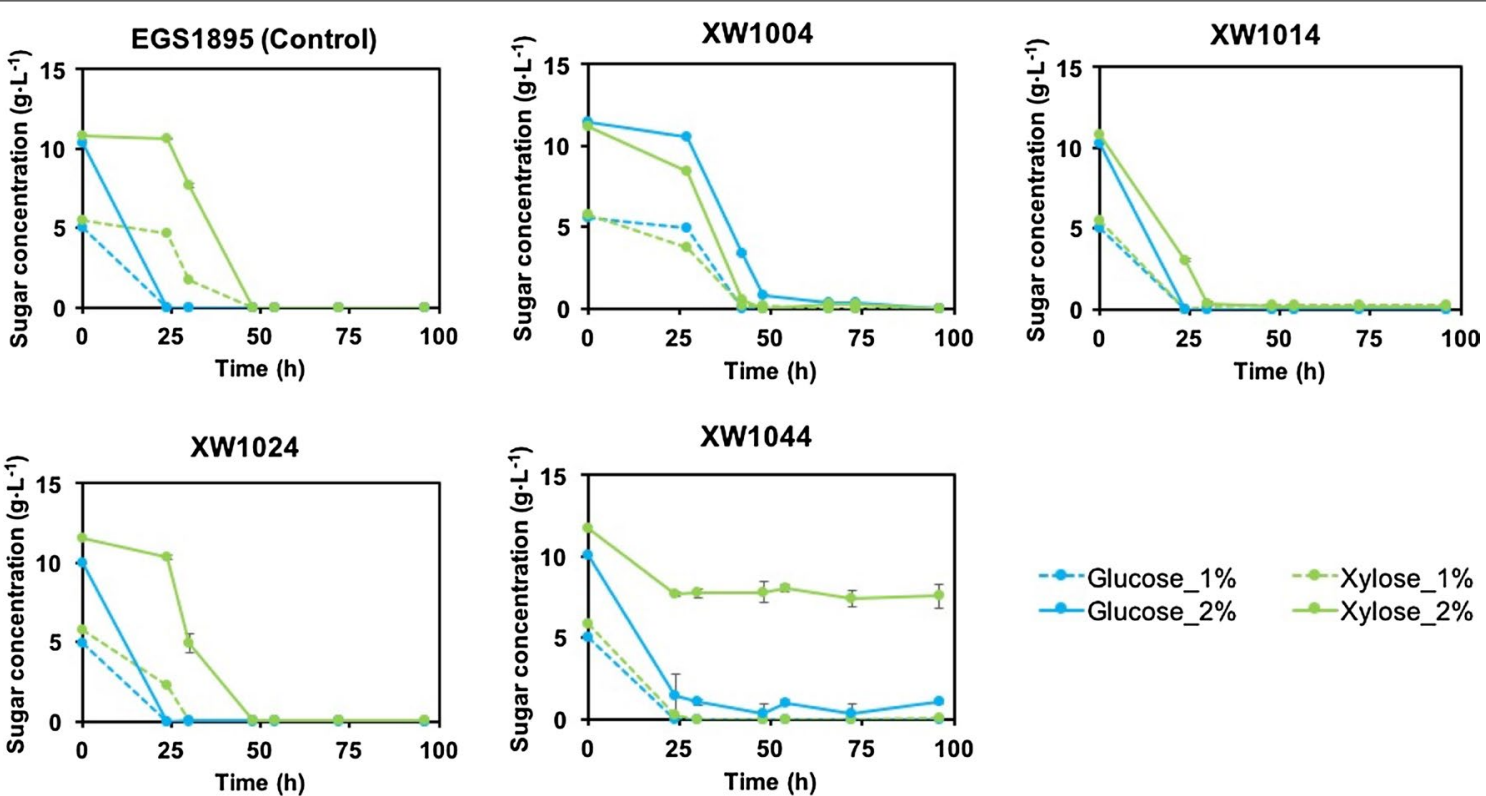

Fig. 1 Evaluation of glucose-xylose co-utilization in engineered strains (96 h). Symbols: glucose, blue lines; xylose, green lines; $1 \%$ total sugars, dashed lines; $2 \%$ total sugars, solid lines. Error bars indicate one standard deviation $(n=3$, except for XW1004, for which $n=2)$ 


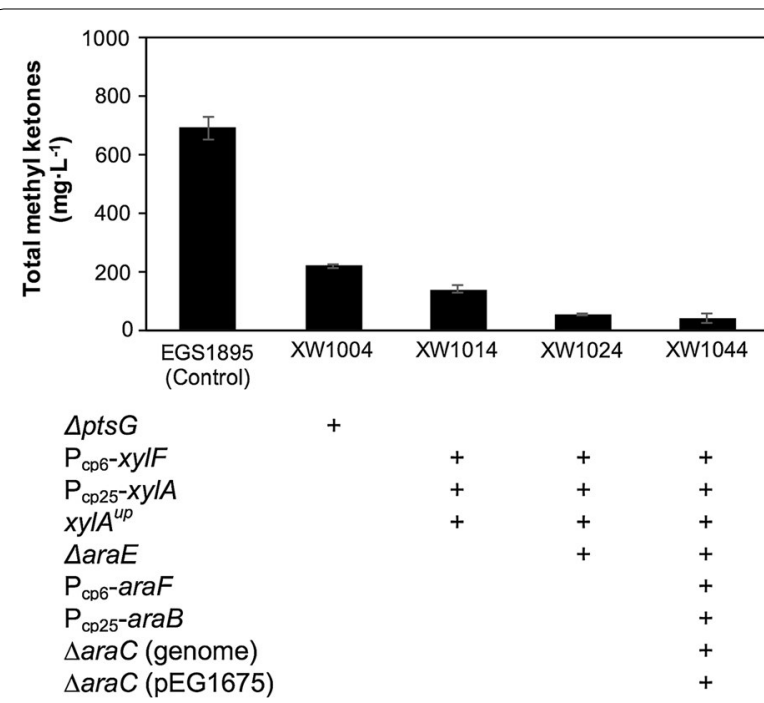

Fig. 2 Methyl ketone production by strains engineered for glucosexylose co-utilization ( $1 \%$ total sugars, 96 h). Error bars indicate one standard deviation ( $n=3$, except for XW1004, for which $n=2$ )

most favorable combination of sugar co-utilization and methyl ketone production of the strains tested.

\section{Optimization of methyl ketone production in strain XW1014 by enhancing NADPH availability}

Although strain XW1014 was successfully engineered for simultaneous glucose-xylose consumption, the significantly reduced methyl ketone titer in this strain necessitated further engineering to improve commercial relevance. We hypothesized that enhancing NADPH availability could be a fruitful engineering target because (1) the biosynthesis of fatty acids (methyl ketone precursors) in E. coli is an NADPH-demanding process and (2) xylose metabolism, particularly when simultaneous with glucose metabolism, could disrupt NADPH production in a host cell (e.g., strain XW1014) compared to conditions with glucose as a sole carbon source. Fatty acid biosynthesis results in net consumption of NADPH due to demand from two key reductases-FabG ( $\beta$-ketoacylACP reductase) and potentially, FabI (enoyl-ACP reductase), which can utilize either $\mathrm{NADH}$ or NADPH as a cofactor [1, 26]. To illustrate the substantial NADPH demands of fatty acid/methyl ketone biosynthesis, production of $1 \mathrm{~mol}$ of a $\mathrm{C}_{13}$ methyl ketone (2-tridecanone) from glucose using the relevant metabolic pathway [2] would result in net consumption of 6 (or 12) mol of $\mathrm{NADPH}$ and net production of 9 (or 15) mol of NADH, depending on FabI cofactor usage.

By virtue of where xylose enters central carbon metabolism in E. coli, xylose metabolism tends to result in less flux than glucose metabolism through the oxidative,
NADPH-generating steps of the pentose phosphate pathway (PPP), namely reactions catalyzed by glucose6-phosphate dehydrogenase (Zwf) and phosphogluconate dehydrogenase (Gnd); however, xylose metabolism can take advantage of other sources of NADPH, such as malic enzyme and transhydrogenase [27]. The situation is likely more complex when considering sugar utilization and NADPH production in strain XW1014 compared to that in control strain EGS1895. Compared with the sequential metabolism from glucose to xylose during diauxic growth (strain EGS1895), simultaneous metabolism of glucose and xylose (strain XW1014) could alter NADPH production by re-distributing flux between glycolysis and the PPP. For example, it is possible that the flux of glucose carbon through the oxidative PPP might be reduced when xylose co-utilization is occurring, because xylose metabolism will satisfy the cell's needs for downstream PPP metabolites required for anabolism, such as erythrose 4-phosphate (needed for aromatic amino acid biosynthesis) and ribose 5-phosphate (needed for nucleic acid biosynthesis).

We implemented two strategies for increasing NADPH supply in strain XW1014: (1) deleting pgi (glucose6-phosphate isomerase) from the chromosome to divert flux from glycolysis through the oxidative PPP (Fig. 3) and (2) overexpressing maeB (malic enzyme), which leads to NADPH generation by oxidative decarboxylation of malate to pyruvate (Fig. 3). ${ }^{13} \mathrm{C}$ Metabolic flux analysis studies in $E$. coli have shown that pgi deletion results in substantial production of NADPH by diversion of flux from glycolysis through the oxidative PPP, and that excessive accumulation of NADPH (cofactor imbalance) in $\Delta p g i$ strains can be at least partially ameliorated by NADPH consumption through transhydrogenase $[28,29]$. In our $\Delta p g i$ strain (XW1054; Table 1), it was anticipated that a portion of the NADPH made available by the pgi deletion might facilitate fatty acid/methyl ketone biosynthesis by better satisfying its high NADPH demands than did central carbon metabolism in strain XW1014.

Production results showed that the $\Delta p g i$ strain (XW1054) had dramatically improved methyl ketone titer $\left(850 \mathrm{mg} \mathrm{L}^{-1}\right.$ ) relative to strain XW1014 after $96 \mathrm{~h}$ at $1 \%$ total sugar conditions (Fig. 4); this methyl ketone titer was comparable to that of the control strain (EGS1895). Under $2 \%$ total sugar conditions, the methyl ketone titer of strain XW1054 ( $1300 \mathrm{mg} \mathrm{L}^{-1}$ after $\left.96 \mathrm{~h}\right)$ was also comparable to that of strain EGS1895 $\left(\sim 1600 \mathrm{mg} \mathrm{L}^{-1}\right)$. However, xylose showed a slower consumption rate than glucose after pgi was deleted, and slower cell growth was also observed during production. In contrast to methyl ketone titer improvement for strain XW1054, the overexpression of $m a e B$ with or without pgi deletion 


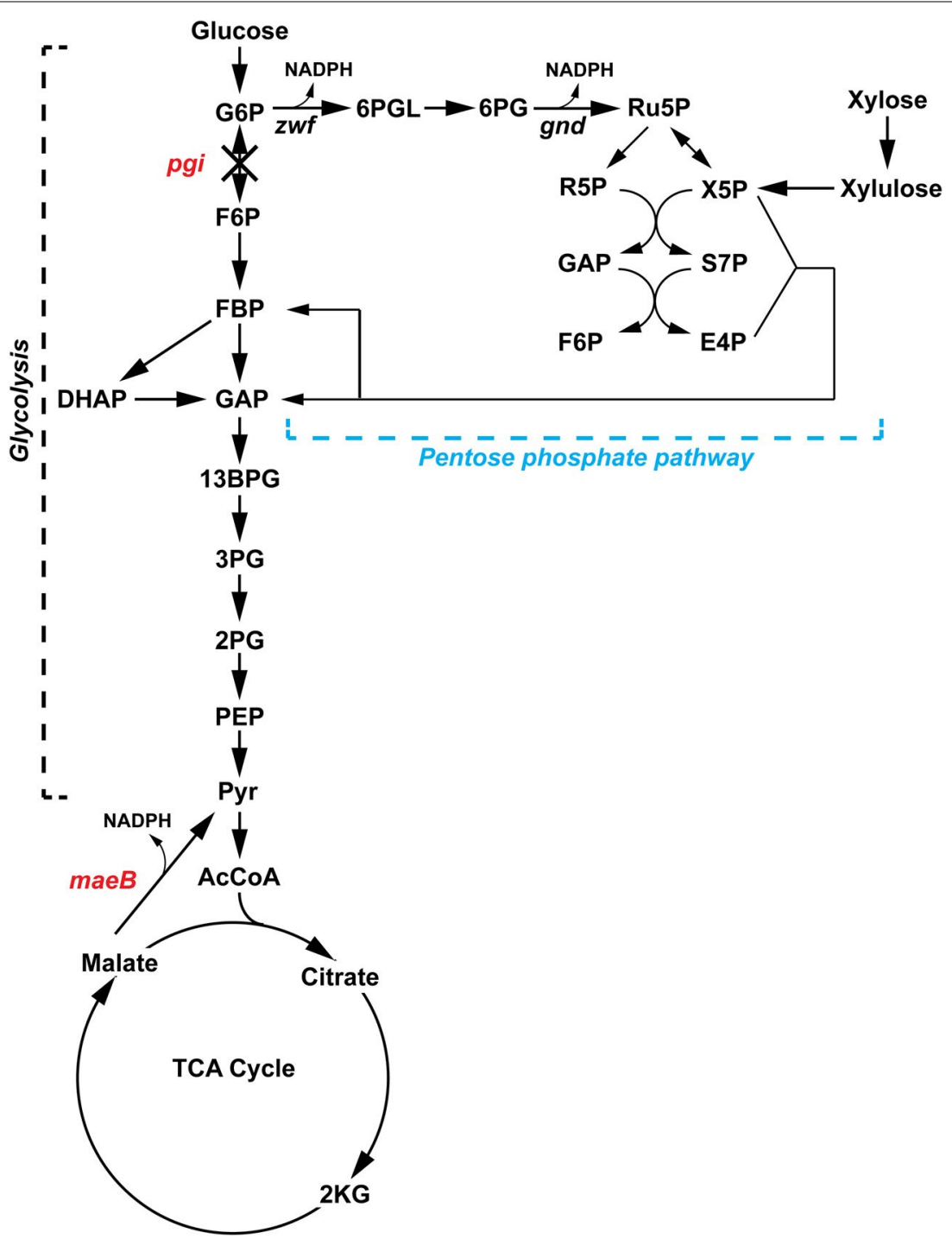

Fig. 3 Overview of central carbon metabolism in E. coli highlighting strategies (in red) to improve NADPH supply in the sugar co-utilizing strain XW1014. G6P, glucose 6-phosphate; F6P, fructose 6-phosphate; FBP, fructose 1,6-bisphosphate; DHAP, dihydroxyacetone phosphate; GAP, glyceraldehyde 3-phosphate; 13BPG, 1,3-bisphosphoglycerate; 3PG, 3-phosphoglycerate; 2PG, 2-phosphoglycerate; PEP, phosphoenolpyruvate; Pyr, pyruvate; AcCoA, acetyl-CoA; 2KG: 2-ketoglutaric acid; 6PGL, 6-phosphogluconolactone; 6PG, 6-phosphogluconate; Ru5P, ribulose 5-phosphate; R5P, ribose 5-phosphate; X5P, xylulose 5-phosphate; S7P, sedoheptulose 7-phosphate; E4P, erythrose 4-phosphate; pgi, glucose 6-phosphate isomerase; maeB, malic enzyme; zwf, glucose 6-phosphate dehydrogenase; gnd, phosphogluconate dehydrogenase

(strains XW1055 and XW1018; Table 1) did not result in improvement in methyl ketone production (Additional file 1: Figure S1).

Based upon the results for strain XW1054 $(\Delta p g i)$, it is possible that NADPH is more limiting when xylose is used as a carbon source. Indeed, we observed that the control strain (EGS1895) produced very low methyl ketone titers when xylose was used as the sole carbon source in minimal medium (Additional file 1: Figure S2).

\section{Optimization of methyl ketone production in strain XW1014 by mutating the RBS of $c r p$}

While enhancing potential NADPH supply (via pgi deletion) substantially improved methyl ketone production with mixed glucose-xylose medium, several lines of evidence suggested that the engineered strains were experiencing suboptimal sugar utilization (e.g., strain XW1054 in Fig. 4), and potentially, suboptimal methyl ketone production, that had causes beyond NADPH limitation. 

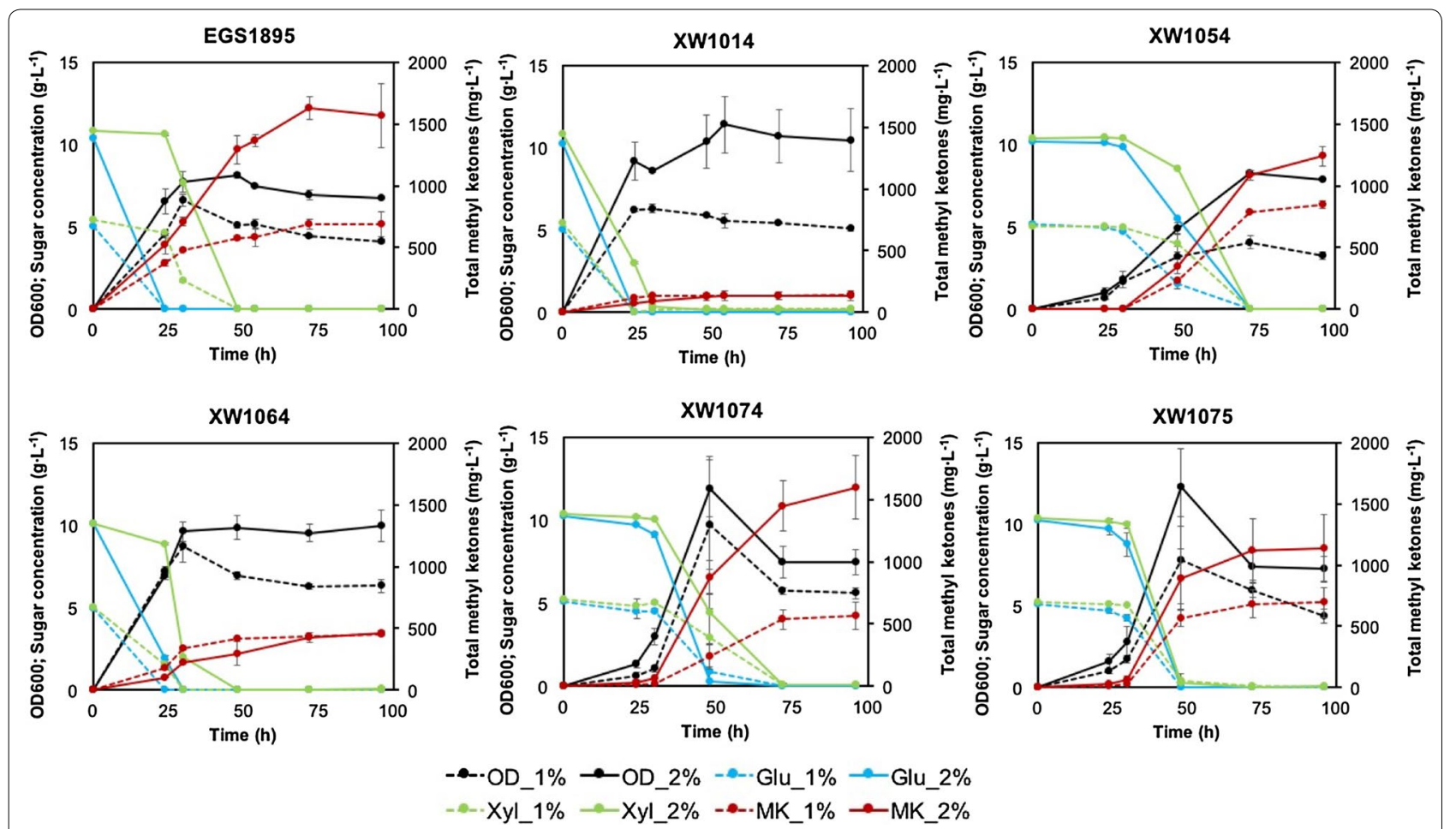

Fig. 4 Shake flask production data (growth, methyl ketone production, sugar consumption) for strains engineered for sugar co-utilization (Table 1) and control strain EGS1895. Symbols: glucose, blue lines; xylose, green lines; $\mathrm{OD}_{600}$, black lines; methyl ketones, red lines; $1 \%$ total sugars, dashed lines; $2 \%$ total sugars, solid lines. The starting $\mathrm{OD}_{600}$ was ca. 0.01 . Error bars indicate one standard deviation $(n=3)$

For example, NADPH limitation alone does not seem to explain the dramatic reduction in methyl ketone titer in both strain XW1004 ( $\Delta p t s G)$ and strain XW1014 (introduced constitutive promoters to $x y l A$ and $x y l F$ ) (Fig. 2), as these genetic modifications are not clearly linked to NADPH supply.

A possible explanation for these results is changes in intracellular distributions of the global regulator CRP. For strain XW1014, promoter replacement for $x y l A$ and $x y l F$ resulted in removal of a CRP binding site from the intergenic region between $x y l A$ and $x y l F$ [30]. As a global regulator, CRP not only plays an important role in carbon catabolite repression, but also controls the transcription of more than 100 genes in E. coli, such as key genes in fatty acid metabolism (e.g., $f a d D, f a d H$ ) [31] and in central carbon metabolism (e.g., pgi, zwf, gnd) [30, 32]. Thus, the promoter change in strain XW1014 might have altered the level of free CRP and directly and indirectly affected the transcription of many other genes related to fatty acid metabolism. Similarly, changes to intracellular CRP pools might also explain why methyl ketone production was reduced in the $\Delta p t s G$ strain (XW1004): the absence of PtsG likely increased cAMP availability [33], and in turn, altered the level of free intracellular CRP, which interacts with cAMP to make the cAMP-CRP complex.

Based on this reasoning, one possible strategy for improving methyl ketone production is to optimize the expression level of CRP in strain XW1014. We attempted to modulate CRP availability by replacing the native $c r p$ RBS with synthetic RBSs of varying strengths. We created a mutant crp RBS library with broad range of predicted TIR values (8-7290 au, Additional file 1: Table S2). A total of 7 RBS variants with different TIRs were identified by sequencing from the mutant library. Screening of this library was conducted with 5-mL cultures in M9-MOPS medium (50-mL test tubes), and one mutant (strain XW1064) was selected that showed significant improvement in methyl ketone production $\left(\sim 900 \mathrm{mg} \mathrm{L}^{-1}\right.$ after $96 \mathrm{~h}$ with $1 \%$ total sugars, Additional file 1: Figure S3). Notably, the predicted TIR of strain XW1064 was $13 \mathrm{au}$, which is approximately 188 -fold lower than the predicted native TIR (2441 au) of $\mathrm{crp}$. Scaled up production of strain XW1064 in 250-mL shake flasks resulted in methyl ketone titers up to $\sim 450 \mathrm{mg} \mathrm{L}^{-1}$ without compromised cell growth (Fig. 4).

This result supported our hypothesis that optimized expression of CRP is able to improve methyl ketone 
production in the strains engineered for glucose-xylose co-utilization. However, we also noticed that the consumption rate of xylose in strain XW1064 was slower than that of glucose, especially under $2 \%$ total sugar conditions (Fig. 4).

\section{Seeking the best candidate by combining engineering strategies}

Given the complementary features of the above strategies ( $\triangle p g i$ and CRP downregulation) on cell growth and methyl ketone production, and the fact that they both effectively improved methyl ketone production in strain XW1014, we decided to combine these two strategies to obtain an additive effect. Overall, combining $\Delta p g i$ and CRP downregulation (strain XW1074; Table 1) created superior phenotypes in cell growth and methyl ketone production compared to use of either strategy alone (Fig. 4). This strain produced up to $570 \mathrm{mg} \mathrm{L}^{-1}$ methyl ketones at $1 \%$ total sugar conditions, but reached a higher titer at $2 \%$ total sugars $\left(\sim 1600 \mathrm{mg} \mathrm{L}^{-1}\right)$ that was comparable to that of the control strain (EGS1895). Glucose and xylose were simultaneously consumed by strain XW1074 (Fig. 4) after a lag period, but utilization of xylose was still slower than that of glucose. Surprisingly, the added maeB overexpression (strain XW1075) dramatically improved sugar co-utilization (albeit with the same lag period, likely caused by pgi deletion; [34, 35]). As a result, strain XW1075 achieved synchronized consumption rates for glucose and xylose at both 1 and $2 \%$ total sugar conditions. Methyl ketone titers in strain XW1075 were up to 700 and $1100 \mathrm{mg} \mathrm{L}^{-1}$ at 1 and $2 \%$ total sugars, respectively. Thus, these two strains engineered with combined strategies (XW1074 and XW1075) represented a favorable phenotype displaying simultaneous utilization of glucose and xylose without substantially sacrificing methyl ketone production relative to the control strain (EGS1895) (Figs. 4, 5).

\section{Strain XW1075 performance during batch fermentation}

Strain XW1075 also compared favorably to control strain EGS1895 in batch fermentation mode. Glucose and xylose were utilized concurrently in strain XW1075 (albeit at unequal rates), whereas strain EGS1895 displayed a typical diauxic pattern, including sequential sugar utilization (Fig. 6). Correspondingly, strain XW1075 had a more consistent methyl ketone production yield (8.7-9.8\%) than the control strain (6.9-10.0\%). At $72 \mathrm{~h}$, the methyl ketone titer of strain XW1075 was $2 \mathrm{~g} \mathrm{~L}^{-1}$, which was ca. $33 \%$ higher than that of strain EGS1895 $\left(1.5 \mathrm{~g} \mathrm{~L}^{-1}\right)$.

Comparison of the results in Fig. 6 with those of strains XW1075 and EGS1895 grown with pure glucose or xylose (Additional file 1: Figure S2) reveals that co-utilization

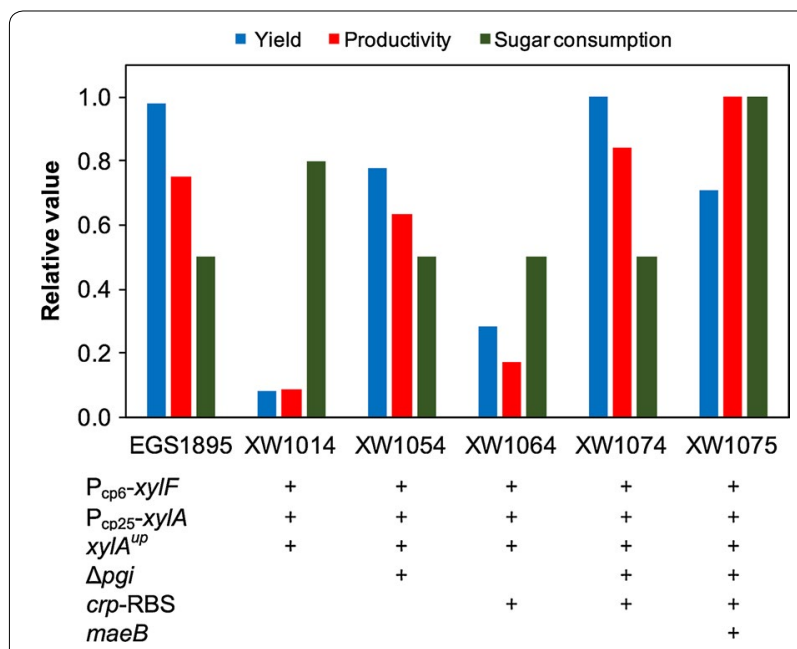

Fig. 5 Summary comparison of methyl ketone production and sugar consumption for engineered strains. Methyl ketone yield, methyl ketone productivity, and sugar consumption period are each normalized to the maximum value among the six strains (for cultivation with $2 \%$ total sugars). Blue, methyl ketone yield from glucose $+x y l o s e$ consumed ( $g$ methyl ketones $\mathrm{g}^{-1}$ total sugars); red, methyl ketone productivity during the sugar consumption period (from onset of sugar consumption to $>90 \%$ total sugar consumption; $\mathrm{g} \mathrm{L}^{-1} \mathrm{~h}^{-1}$ ); green, the reciprocal of sugar consumption period (as defined for productivity; the reciprocal was used to make the most favorable consumption phenotype approach 1 instead of 0 for ease of comparison)

of glucose and xylose in strain XW1075 enabled substantially better methyl ketone production than did utilization of either sugar alone. In fact, methyl ketone production was negligible for strain XW1075 utilizing either pure glucose or pure xylose (Additional file 1: Figure S2). Notably, strain EGS1895 also produced negligible methyl ketones when grown on pure xylose (Additional file 1: Figure S2), but produced substantial methyl ketones while metabolizing xylose after diauxic depletion of glucose (Fig. 6). From Figs. 4 and 6, it appears that glucose metabolism supported both growth and methyl ketone production in strain EGS1895, whereas xylose metabolism supported methyl ketone production but little or no growth.

\section{Conclusions}

In this study, genetic manipulations were conducted to alleviate carbon catabolite repression in our most efficient methyl ketone-producing strain. A strain (XW1014) with constitutively expressed $x y l A$ and $x y l F$ plus a $x y l A$ promoter mutation showed well-synchronized glucose and xylose consumption rates. However, this newly acquired capability came at the expense of methyl ketone titer, which decreased fivefold. Further efforts were made to optimize methyl ketone production in this strain, and 

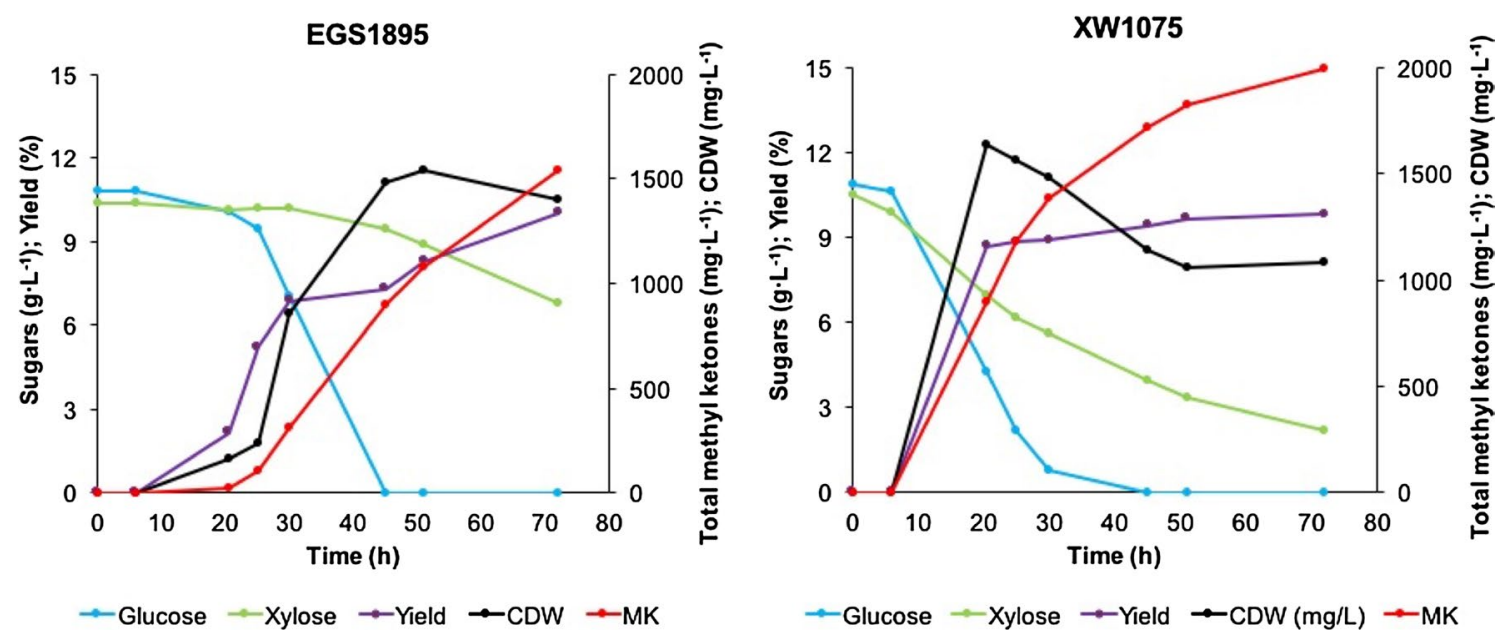

Fig. 6 Batch fermentation of strains EGS1895 and XW1075 in 2-L bioreactors. Symbols: glucose, blue line; xylose, green line; cell dry weight (CDW), black line; methyl ketones, red line; yield, purple line

we found that chromosomal deletion of pgi (to enhance $\mathrm{NADPH}$ supply) and CRP downregulation by replacement of the native RBS both effectively improved methyl ketone production. Combining these strategies resulted in the most favorable overall phenotypes for simultaneous glucose-xylose consumption without compromising methyl ketone titer (Figs. 5, 6). Further optimization of performance will entail improved fermentation process conditions as well as additional genetic modifications.

\section{Additional file}

Additional file 1. Supplementary figures and tables.

\section{Abbreviations}

CCR: carbon catabolite repression; PEP: phosphoenol pyruvate; PTS: phosphotransferase; CAMP: cyclic AMP; CRP: CAMP receptor protein; PPP: pentose phosphate pathway; RBS: ribosomal binding site; TIR: translation initiation rate; MK: methyl ketones; CDW: cell dry weight.

\section{Authors' contributions}

$H B$ and XW designed the experiments. XW developed strains and performed shake flask production. EG developed the bioreactor fermentation method and $E G$ and XW performed the fermentation. XW, HB and EG analyzed the data. XW, HB, and EG wrote the manuscript. All authors read and approved the final manuscript.

\section{Author details}

1 Joint BioEnergy Institute (JBEI), 5885 Hollis St., Emeryville, CA 94608, USA. ${ }^{2}$ Biological Systems \& Engineering Division, Lawrence Berkeley National Laboratory, Berkeley, CA 94720, USA. ${ }^{3}$ Earth \& Environmental Sciences, Lawrence Berkeley National Laboratory, Berkeley, CA 94720, USA.

\section{Acknowledgements}

We thank Jeremy Minty (Ecovia Renewables, Inc.), Sung Kuk Lee (Ulsan National Institute of Science and Technology, Korea), and Paul Opgenorth and Joonhoon Kim (Joint BioEnergy Institute) for valuable discussions.

\section{Competing interests}

The authors declare that they have no competing interests.

\section{Availability of data and materials}

All data generated or analyzed during this study are included in this published article and its additional files.

\section{Consent for publication}

Not applicable.

Ethics approval and consent to participate

Not applicable.

\section{Funding}

This work was supported by U.S. Department of Energy (DOE) Phase I Small Business Technology Transfer (STTR) project DE-SC0015093. The portion of the work conducted at the Joint BioEnergy Institute (JBEI) was supported by the Office of Science, Office of Biological and Environmental Research, of the DOE under Contract No. DE-AC02-05CH11231.

\section{Publisher's Note}

Springer Nature remains neutral with regard to jurisdictional claims in published maps and institutional affiliations.

Received: 10 September 2017 Accepted: 19 January 2018

Published online: 27 January 2018

\section{References}

1. Beller HR, Lee TS, Katz L. Natural products as biofuels and bio-based chemicals: fatty acids and isoprenoids. Nat Prod Rep. 2015;32:1508-26.

2. Goh E-B, Baidoo EEK, Burd H, Lee TS, Keasling JD, Beller HR. Substantial improvements in methyl ketone production in E. coli and insights on the pathway from in vitro studies. Metab Eng. 2014;26:67-76.

3. Goh E-B, Baidoo EEK, Keasling JD, Beller HR. Engineering of bacterial methyl ketone synthesis for biofuels. Appl Environ Microbiol. 2012;78:70-80.

4. Lennen RM, Pfleger BF. Microbial production of fatty acid-derived fuels and chemicals. Curr Opin Biotechnol. 2013;24:1044-53. 
5. Park J, Rodríguez-Moyá M, Li M, Pichersky E, San K-Y, Gonzalez R. Synthesis of methyl ketones by metabolically engineered Escherichia coli. J Ind Microbiol Biotechnol. 2012;39:1703-12.

6. Kim J-H, Block DE, Mills DA. Simultaneous consumption of pentose and hexose sugars: an optimal microbial phenotype for efficient fermentation of lignocellulosic biomass. Appl Microbiol Biotechnol. 2010;88:1077-85.

7. Deutscher J. The mechanisms of carbon catabolite repression in bacteria. Curr Opin Microbiol. 2008;11:87-93.

8. Deutscher J, Francke C, Postma PW. How phosphotransferase systemrelated protein phosphorylation regulates carbohydrate metabolism in bacteria. Microbiol Mol Biol Rev. 2006;70:939-1031.

9. Desai TA, Rao CV. Regulation of arabinose and xylose metabolism in Escherichia coli. Appl Environ Microbiol. 2010;76:1524-32.

10. Nichols N, Dien B, Bothast R. Use of catabolite repression mutants for fermentation of sugar mixtures to ethanol. Appl Microbiol Biotechnol. 2001;56:120-5.

11. Yao R, Hirose Y, Sarkar D, Nakahigashi K, Ye Q, Shimizu K. Catabolic regulation analysis of Escherichia coli and its $c r p, m / c, m g s A$, pgi and pts $G$ mutants. Microb Cell Fact. 2011;10:67.

12. Kim SM, Choi BY, Ryu YS, Jung SH, Park JM, Kim G-H, Lee SK. Simultaneous utilization of glucose and xylose via novel mechanisms in engineered Escherichia coli. Metab Eng. 2015:30:141-8.

13. Xia T, Eiteman MA, Altman E. Simultaneous utilization of glucose, xylose and arabinose in the presence of acetate by a consortium of Escherichia coli strains. Microb Cell Fact. 2012;11:77.

14. Chiang C-J, Lee HM, Guo HJ, Wang ZW, Lin L-J, Chao Y-P. Systematic approach to engineer Escherichia coli pathways for co-utilization of a glucose-xylose mixture. J Agric Food Chem. 2013;61:7583-90.

15. Groff D, Benke PI, Batth TS, Bokinsky G, Petzold CJ, Adams PD, Keasling JD. Supplementation of intracellular XyIR leads to coutilization of hemicellulose sugars. Appl Environ Microbiol. 2012;78:2221-9.

16. Wu Y, Shen X, Yuan $Q$, Yan Y. Metabolic engineering strategies for coutilization of carbon sources in microbes. Bioengineering. 2016;3:10

17. San K-Y, Li M, Zhang X. Bacteria and method for synthesizing fatty acids. US Patent 2016. US9309543B2.

18. Qiao K, Wasylenko TM, Zhou K, Xu P, Stephanopoulos G. Lipid production in Yarrowia lipolytica is maximized by engineering cytosolic redox metabolism. Nat Biotechnol. 2017:35:173-7.

19. Datsenko KA, Wanner BL. One-step inactivation of chromosomal genes in Escherichia coli K-12 using PCR products. Proc Natl Acad Sci USA. 2000;97:6640-5

20. Baba T, Ara T, Hasegawa M, Takai Y, Okumura Y, Baba M, Datsenko KA, Tomita M, Wanner BL, Mori H. Construction of Escherichia coli K-12 inframe, single-gene knockout mutants: the Keio collection. Mol Syst Biol. 2006;2:2006.0008

21. Jensen PR, Hammer K. The sequence of spacers between the consensus sequences modulates the strength of prokaryotic promoters. Appl Environ Microbiol. 1998;64:82-7.

22. Farasat I, Kushwaha M, Collens J, Easterbrook M, Guido M, Salis HM. Efficient search, mapping, and optimization of multi-protein genetic systems in diverse bacteria. Mol Syst Biol. 2014;10:731.
23. Espah Borujeni A, Channarasappa AS, Salis HM. Translation rate is controlled by coupled trade-offs between site accessibility, selective RNA unfolding and sliding at upstream standby sites. Nucleic Acids Res. 2014;42:2646-59.

24. Salis HM, Mirsky EA, Voigt CA. Automated design of synthetic ribosome binding sites to control protein expression. Nat Biotechnol. 2009;27:946-50.

25. Korz DJ, Rinas U, Hellmuth K, Sanders EA, Deckwer WD. Simple fed-batch technique for high cell density cultivation of Escherichia coli. J Biotechnol. 1995;39:59-65.

26. Bergler H, Fuchsbichler S, Högenauer G, Turnowsky F. The enoyl-[acylcarrier-protein] reductase (Fabl) of Escherichia coli, which catalyzes a key regulatory step in fatty acid biosynthesis, accepts NADH and NADPH as cofactors and is inhibited by palmitoyl-CoA. Eur J Biochem. 1996;242:689-94.

27. Gonzalez JE, Long CP, Antoniewicz MR. Comprehensive analysis of glucose and xylose metabolism in Escherichia coli under aerobic and anaerobic conditions by ${ }^{13} \mathrm{C}$ metabolic flux analysis. Metab Eng. 2017;39:9-18.

28. Hua Q, Yang C, Baba T, Mori H, Shimizu K. Responses of the central metabolism in Escherichia coli to phosphoglucose isomerase and glucose6-phosphate dehydrogenase knockouts. J Bacteriol. 2003;185:7053-67.

29. Canonaco F, Hess TA, Heri S, Wang T, Szyperski T, Sauer U. Metabolic flux response to phosphoglucose isomerase knock-out in Escherichia coli and impact of overexpression of the soluble transhydrogenase UdhA. FEMS Microbiol Lett. 2001;204:247-52.

30. Shimada T, Fujita N, Yamamoto K, Ishihama A. Novel roles of cAMP receptor protein (CRP) in regulation of transport and metabolism of carbon sources. PLoS ONE. 2011;6:e20081.

31. Gama-Castro S, Salgado H, Santos-Zavaleta A, Ledezma-Tejeida D, MuñizRascado L, García-Sotelo JS, Alquicira-Hernández K, Martínez-Flores I, Pannier L, Castro-Mondragón JA, et al. RegulonDB version 9.0: highlevel integration of gene regulation, coexpression, motif clustering and beyond. Nucleic Acids Res. 2016;44:D133-43.

32. Zheng D, Constantinidou C, Hobman JL, Minchin SD. Identification of the CRP regulon using in vitro and in vivo transcriptional profiling. Nucleic Acids Res. 2004;32:5874-93.

33. Steinsiek S, Bettenbrock K. Glucose transport in Escherichia coli mutant strains with defects in sugar transport systems. J Bacteriol. 2012;194:5897-908.

34. Toya Y, Ishii N, Nakahigashi K, Hirasawa T, Soga T, Tomita M, Shimizu K. ${ }^{13} \mathrm{C}$-metabolic flux analysis for batch culture of Escherichia coli and its pyk and pgi gene knockout mutants based on mass isotopomer distribution of intracellular metabolites. Biotechnol Prog. 2010;26:975-92.

35. Kabir MM, Shimizu K. Gene expression patterns for metabolic pathway in pgi knockout Escherichia coli with and without phb genes based on RT-PCR. J Biotechnol. 2003;105:11-31.

\section{Submit your next manuscript to BioMed Central and we will help you at every step:}

- We accept pre-submission inquiries

- Our selector tool helps you to find the most relevant journal

- We provide round the clock customer support

- Convenient online submission

- Thorough peer review

- Inclusion in PubMed and all major indexing services

- Maximum visibility for your research

Submit your manuscript at www.biomedcentral.com/submit
BioMed Central 